\title{
The Clinical Features of Ischemic Stroke Patients for Whom Smoking Was Considered the Sole Risk Factor for Ischemic Stroke
}

Takuya Fukuoka, Yoshihiko Nakazato, Hitoshi Kawasaki, Kei Ikeda, Tomotsugu Furuya, Akifumi Miyake, Takashi Mitsufuji, Yasuo Ito, Kazushi Takahashi, Nobuo Araki, Norio Tahahashi and Toshimasa Yamamoto

\begin{abstract}
:
Objective The purpose of this study was to clarify the clinical features of ischemic patients for whom cigarette smoking was the sole risk factor for ischemic stroke.

Methods Among the 1,329 patients (male, $n=833$; female, $n=496$ ) with acute ischemic stroke who were admitted to our hospital between April 2005 and September 2016, 346 (26\%) were smokers [male, n=308 (36.9\%); female, $38(7.6 \%)]$. In 42 (3.1\%; male, $\mathrm{n}=41$; female, $\mathrm{n}=1)$ cases, cigarette smoking was considered to be the sole risk factor for ischemic stroke. Data regarding gender, age, the clinical type of ischemic stroke, the National Institutes of Health Stroke Scale (NIHSS) score at the admission, the modified Rankin scale (mRS) scores before the onset and at discharge, the progression of symptoms, and the recurrence of infarction were investigated.

Results The mean age of the 42 patients was $63.2 \pm 12.4$ years (range, 26-86 years). The clinical types of ischemic stroke included atherothrombosis $(n=19)$, lacunar $(n=17)$, other type $(n=2)$ and undetermined type ( =4). The median NIHSS score at the time of admission for ischemic stroke was 2 (interquartile range: IQR 1-4.25). The median mRS scores before the onset and at the discharge were 0 (IQR 0-0) and 1 (IQR 0-2), respectively. One patient had symptoms of progression; no patients had recurrence of infarction.

Conclusion Our findings suggest that cigarette smoking alone may induce ischemic stroke; moreover, patients for whom smoking was the sole risk factor for ischemic stroke showed milder symptoms in comparison to patients with other risk factors; however, ischemic stroke was induced from youth. Since cigarette smoking has detrimental effects on the central nervous system, we suggest that people be encouraged to quit smoking in order to maintain good health.
\end{abstract}

Key words: cigarette smoking, ischemic stroke

(Intern Med 57: 1703-1706, 2018)

(DOI: 10.2169/internalmedicine.9655-17)

\section{Introduction}

Cigarette smoking is a risk factor for ischemic stroke (1), and smoking is a well-known independent risk factor for myocardial infarction and stroke (2). However, the characteristics of ischemic stroke patients for whom cigarette smoking is the sole risk factor for ischemic stroke have not been fully investigated. This study aimed to clarify these characteristics.

\section{Materials and Methods}

Among the 1,329 patients (male, $\mathrm{n}=833$; female, $\mathrm{n}=496$ ) with acute ischemic stroke admitted at our hospital between April 2005 and September 2016, 346 (26\%) were smokers 
Table 1. Comparison of Risk Factors Except for Cigarette Smoking in Ischemic Stroke Patients Classified by Smoking and Non Smoking.

\begin{tabular}{lccc}
\hline & Nonsmoking group $(\mathrm{n}=983)$ & Smoking group $(\mathrm{n}=346)$ & $\mathrm{p}$ value \\
\hline Hypertension \% (n) & $68.2(670)$ & $67.6(234)$ & $\mathrm{ns}$ \\
Diabetes mellitus \% (n) & $29.2(287)$ & $35.0(121)$ & $<0.05$ \\
Dyslipidemia \% (n) & $35.8(352)$ & $43.6(151)$ & $<0.05$ \\
Atrial fibrillation \% (n) & $20.1(198)$ & $12.1(42)$ & $<0.05$ \\
Coagulation disorder \% (n) & $0.2(2)$ & $0.6(2)$ & $\mathrm{ns}$ \\
Chronic renal failure \% (n) & $12.5(123)$ & $8.1(28)$ & $<0.05$ \\
Obesity \% (n) & $29.7(292)$ & $29.8(103)$ & $\mathrm{ns}$ \\
Sleep apnea syndrome \% (n) & $0(0)$ & $0(0)$ & $\mathrm{ns}$ \\
Ischemic heart disease \% (n) & $6.2(61)$ & $5.2(18)$ & $\mathrm{ns}$ \\
\hline
\end{tabular}

n: number, ns: no significant difference

[male, $\mathrm{n}=308$ (36.9\%); female, 38 (7.6\%)]. In 42 cases $(3.1 \%)$, cigarette smoking was considered to be the sole risk factor for ischemic stroke. Data regarding gender, age, clinical type of ischemic stroke, National Institutes of Health Stroke Scale (NIHSS) score at admission, modified Rankin scale (mRS) score before the onset and at discharge, symptom progression, and recurrence of infarction were investigated. Symptom progression was defined as a worsening of the NIHSS score by $\geq 1$. Recurrence of ischemic stroke was defined as the detection of another area of infarction on brain MRI. In this study, patients were classified as smokers if they smoked at least 1 cigarette/day for $>1$ year. Never smokers and passive smokers who had stopped smoking within 1 year before the index stroke were classified as nonsmokers.

None of the 42 patients in the smoking alone group showed hypertension, dyslipidemia, diabetes mellitus, atrial fibrillation, coagulation disorder, chronic renal failure, obesity (body mass index $>25 \mathrm{~kg} / \mathrm{m}^{2}$ ), or sleep apnea syndrome, either in their clinical history or at admission.

The following risk factors were defined according to Japanese guidelines: systolic blood pressure $\geq 140 \mathrm{~mm} \mathrm{Hg}$ or diastolic blood pressure $\geq 90 \mathrm{~mm} \mathrm{Hg}$ for hypertension (3); total cholesterol $\geq 220 \mathrm{mg} / \mathrm{dL}$, low-density lipoprotein cholesterol $\geq 140 \mathrm{mg} / \mathrm{dL}$, high-density lipoprotein cholesterol $\leq 40 \mathrm{mg} / \mathrm{dL}$, or triglyceride $\geq 150 \mathrm{mg} / \mathrm{dL}$ for dyslipidemia (4); and fasting plasma glucose $\geq 126 \mathrm{mg} / \mathrm{dL}$, any blood glucose $\geq 200 \mathrm{mg} / \mathrm{dL}$ in the 75 -g glucose tolerance test, or hemoglobin A1c $\geq 6.5 \%$ for diabetes mellitus (5).

The clinical types were classified in accordance with the clinical categories of the National Institute of Neurological Disorders and Stroke (NINDS) (6). However, as noted above, cigarette smoking was the only risk factor for ischemic stroke among the 42 patients in the smoking alone group. Thus, the following clinical types of ischemic stroke in the smoking alone group were classified into the following types, based on the magnetic resonance imaging findings and the clinical course: atherothrombosis, lacunar, other type and undetermined type. In patients with lacunar infarcts, brain imaging showed a small lesion $(<1.5 \mathrm{~cm}$ at its greatest diameter) in a location compatible with the deficit.
Atherothrombosis infarction occurs with atherosclerosis involving selected sites in the extracranial and major intracranial arteries (6). The undetermined type does not fall into any of these categories.

Two authors independently assessed the clinical types of ischemic stroke. If their diagnoses differed, the results were discussed until they reached a consensus.

\section{Statistical analysis}

The statistical analyses were performed using the PASW Statistics software program (version 18, SPSS Science, Chicago, USA). The NIHSS score at admission, and the mRS before the onset and at discharge were compared using the Kruskal-Wallis test with Bonferroni correction. Ratios were compared using the chi-squared test. In all of the analyses, a $\mathrm{p}$ value of $<0.05$ was considered to indicate statistical significance. The present study was approved by the Ethics Committee of Saitama Medical University (application no. 17-012).

\section{Results}

Table 1 demonstrates the risk factors of the smoking alone group and the nonsmoking group in the present study.

The clinical features of the 42 patients (male, $n=41$; female, $n=1$ ) are shown in Table 2 . The mean ( \pm standard deviation) age was $63.2 \pm 12.4$ years (range, 26-86 years). The clinical types of ischemic stroke included atherothrombosis $(n=19)$, lacunar $(n=17)$, and other type $(n=2)$ and undetermined $(n=4)$. The median NIHSS score at the time of admission for ischemic stroke was 2 (interquartile range: IQR 1-4.25). The median mRS scores before the onset and at discharge were 0 (IQR 0-0) and 1 (IQR 0-2) respectively. One patient had symptom progression; no patients had recurrence of infarction. The age of the smoking alone group was younger and the NIHSS scores at admission, and the mRS scores at discharge were lower in comparison to the other groups. 
Table 2. Comparison of Gender, Age and Prognosis in Ischemic Stroke Patients Classified by Presence of Other Risk Factor with Smoking and Non Smoking.

\begin{tabular}{|c|c|c|c|c|c|}
\hline \multirow{2}{*}{ Other risk factor ${ }^{\dagger}$} & \multicolumn{2}{|c|}{ Non smoking group $(\mathrm{n}=983)$} & \multicolumn{2}{|c|}{ Smoking group $(\mathrm{n}=346)$} & \multirow{2}{*}{$\mathrm{p}$ value } \\
\hline & yes $(n=859)$ & no $(n=124)$ & yes $(n=304)$ & no $(n=42)$ & \\
\hline Gender & M: 459, F: 400 & M: 66, F: 58 & M: 267, F: 37 & M: 41, F: 1 & \\
\hline Age & $71.5 \pm 11.3$ & $68.7 \pm 16.6$ & $64.3 \pm 11$ & $62.3 \pm 12.4$ & \\
\hline NIHSS at admission median (IQR) & $4(2-8)^{*}$ & $4(1-6)$ & $3(2-7)$ & $2(1-4.25)$ & $<0.05$ \\
\hline mRS before the onset median (IQR) & $0(0-1)$ & $0(0-1)$ & $0(0-0)$ & $0(0-0)$ & ns \\
\hline $\mathrm{mRS}$ at discharge median (IQR) & $2(1-4)^{*}$ & $1(0-3)$ & $1(1-2)$ & $1(0-2)$ & $<0.05$ \\
\hline Symptom progression $\%(\mathrm{n})$ & $12(103)$ & $10.5(13)$ & $10.5(32)$ & $2.4(1)$ & $\mathrm{ns}$ \\
\hline Recurrence of ischemic stroke \% (n) & $2.9(25)$ & $4.8(6)$ & $5.3(16)$ & $0(0)$ & ns \\
\hline Clinical type \%(n) & $\begin{array}{l}\text { A: } 46.2(397) \\
\text { L: } 29.2(252) \\
\text { C: } 21.3(183) \\
\text { O: } 3.1(27)\end{array}$ & $\begin{array}{l}\text { A: } 43.5(54) \\
\text { L: } 29(36) \\
\text { O: } 10.5(13) \\
\text { U: } 16.9(21)\end{array}$ & $\begin{array}{l}\text { A: } 43.8(133) \\
\text { L: } 35.9(109) \\
\text { C: } 17.8(54) \\
\text { O: } 2.6(8)\end{array}$ & $\begin{array}{l}\text { A: } 45.2(19) \\
\text { L: } 40.5(17) \\
\text { O: } 4.8(2) \\
\text { U: } 9.5(4)\end{array}$ & \\
\hline
\end{tabular}

$\dagger$ Other risk factor is hypertension, diabetes mellitus, dyslipidemia, atrial fibrillation, any coagulation disorder, chronic renal failure, obesity and sleep apnea syndrome.

* relative to the corresponding smoking alone as ischemic stroke risk factor and no other risk factors.

M: male, F: female, A: atherothrombosis, L: lacunar, C: cardiogenic embolism, O: other type, U: undetermined type, ns: no significant difference, IQR: interquartile range, NIHSS: National Institutes of Health Stroke Scale, mRS: modified Rankin scale

\section{Discussion}

Cigarette smoking is regarded as a "silent killer" and a risk factor for stroke (1). No previous reports have investigated the clinical features of ischemic stroke in patients in whom cigarette smoking was considered to be the sole risk factor. Regarding gender bias, Kubo et al. reported that the rate of ischemic infarction in men tended to be higher than that in women (1.4-1.8 odds) (7). In the present study the incidence of ischemic stroke in men in whom cigarette smoking was considered to be the sole risk factor was higher than that in women. We hypothesized that the arteriosclerotic changes and cardiac load in men are greater in comparison to those in women. Moreover, the patients in the smoking alone group were younger at the onset of ischemic stroke. This may be due to the fact that many of the cigarette smokers had smoked since young adulthood without hypertension, dyslipidemia, diabetes mellitus, or atrial fibrillation. Arteriosclerosis and cardiac loading may start at a young age. Actually, the smoking rate in Japan is high in individuals who range from their thirties to fifties, and decreases among individuals in their sixties (8). Moreover, since smoking has been associated with increased hematocrit levels, platelet activation and aggregation, vasoconstriction, increased circulating levels of fibrinogen, thrombin generation and an impaired endogenous fibrinolytic capacity (9-11) - in smokers - vascular occlusion may be induced by thrombogenic factors rather than atherogenic factors. Thus, it is expected that smoking-associated ischemic infarction may occur at a younger age. However, we need to consider that the smoking population in Japan is comprised of younger individuals. The younger age of the patients was considered to be the reason why the NIHSS scores at admission and the mRS scores at discharge were lower in the smoking group.
Thus, we considered that their younger age would have been associated with a good recovery. However, in addition to causing stroke and major coronary events (7), cigarette smoking also causes arrhythmia $(12,13)$. The patients had no history of atrial fibrillation and atrial fibrillation was not detected by repeated Holter monitoring on admission. However, this does not preclude the possibility of cigarette smoking-induced arrhythmias.

\section{Conclusion}

Patients in the smoking alone group showed milder symptoms in comparison to other groups; however, ischemic stroke was induced from youth. The results of the present study suggests that cigarette smoking has the potential to cause stroke and that it is necessary to discourage smoking for the preservation of health.

The authors state that they have no Conflict of Interest (COI).

\section{References}

1. O’Donnell MJ, Chin SL, Rangarajan S, et al. Global and regional effects of potentially modifiable risk factors associated with acute stroke in 32 countries (INTERSTROKE): a case-control study. Lancet 388: 761-775, 2016.

2. Bartecchi CE, MacKenzie TD, Schrier RW. The human costs of tobacco use (1). N Engl J Med 330: 907-912, 1994.

3. Japanese Society of Hypertension guidelines for the management of hypertension (JSH 2004). Hypertens Res 29 (Suppl): S1-S105, 2006.

4. Japan Atherosclerosis Society. Japan Atherosclerosis Society (JAS) guidelines for diagnosis and treatment of atherosclerotic cardiovascular diseases, 2014 [Internet]. [cited 2017 Sep. 18]. Available from: https://www.jstage.jst.go.jp/article/jat/20/8/20_17152/_pdf

5. The Japan Diabetes Society. Evidence-Based Practice Guideline for the Treatment of Diabetes in Japan. Nankodo, Tokyo, 2016 (in 
Japanese)

6. Special report from the National Institute of Neurological Disorders and Stroke. Classification of cerebrovascular diseases III. Stroke 21: 637-676, 1990.

7. Kubo M, Kiyohara Y, Kato I, et al. Trends in the incidence, mortality, and survival rate of cardiovascular disease in a Japanese community: the Hisayama study. Stroke 34: 2349-2354, 2003.

8. Ministry of Health LaWW. The National Health and Nutrition Survey in Japan, 2015 (in Japanese) [Internet]. [cited 2017 Sep. 18]. Available from: http://www.mhlw.go.jp/bunya/kenkou/eiyou/dl/h27houkoku-07.pdf

9. Purcell IF, Newall N, Farrer M. Lower cardiac mortality in smokers following thrombolysis for acute myocardial infarction may be related to more effective fibrinolysis. QJM 92: 327-333, 1999.

10. Zidovetzki R, Chen P, Fisher M, Hofman FM, Faraci FM. Nico- tine increases plasminogen activator inhibitor-1 production by human brain endothelial cells via protein kinase C-associated pathway. Stroke 30: 651-655, 1999.

11. McGill HC Jr. The cardiovascular pathology of smoking. Am Heart J 115: 250-257, 1988.

12. Heeringa J, Kors JA, Hofman A, van Rooij FJ, Witteman JC. Cigarette smoking and risk of atrial fibrillation: the Rotterdam Study. Am Heart J 156: 1163-1169, 2008.

13. Stewart PM, Catterall JR. Chronic nicotine ingestion and atrial fibrillation. Br Heart J 54: 222-223, 1985.

The Internal Medicine is an Open Access article distributed under the Creative Commons Attribution-NonCommercial-NoDerivatives 4.0 International License. To view the details of this license, please visit (https://creativecommons.org/licenses/ by-nc-nd/4.0/).

(C) 2018 The Japanese Society of Internal Medicine Intern Med 57: 1703-1706, 2018 